\title{
ベイジアンネットワークによる回転機械の状態診断法
}

\section{Diagnosis method for Rotating Machinery Using Bayesian Network}

\author{
非 朱晶晶（三重大） \\ 非李可 (三重大) \\ 非 薛紅濤 (三重大) \\ ○正 陳山鵬（三重大）
}

Jingjing Zhu, Mie University

Ke Li, Mie University

Hongtao Xue, Mie University

Ho JINYAMA, Mie University, 1577 Kurimamachiyacho-cho, Tsu

In order to effectively identify faults of a rotating mechanics, a new kind of symptom parameter---.--Relative Ratio Symptom Parameter (RRSP) is proposed in this paper. Moreover, combined with Bayesian Network, the corresponding fault diagnosis system is built. In the paper, the vibration signals are monitored and measured and the relative ratio symptom parameter is calculated, of which the parameters whose identification index is bigger are chosen as the input of Bayesian Network, by observing and analyzing the output that is the probability of normal state and abnormal states, Bayesian Network in the mechanical fault diagnosis is proved to be effective by real data measured in each state of a rotating machine.

Key Words: Bayesian Network, Rotating machine, Symptom parameter, Fault diagnosis

\section{INTRODUCTION}

With the development of the intelligence and complexity in the rotating machinery, the monitoring and fault diagnosis for rotating machinery are becoming more and more important to prevent accidents and financial loss. Furthermore, when developing intelligent condition diagnosis system by computer, symptom parameters (SPs) are required to express the feature of vibration signals measured for distinguishing machine states. If we can find good symptom parameters which sensitively reflect the feature of the machine states, automatic diagnosis for machine faults is possible ${ }^{[1]}$. However, in many cases of the condition diagnosis for rotating machinery, especially in an early stage of a fault, the effect of noise in the signal measured for the diagnosis is so strong that the symptom of the fault is not evident and there are a lot of ambiguous relationships between symptom parameters and failure types of plant machinery, so failure types of plant machinery cannot be easily identified ${ }^{[2][3][4]}$.

In order to resolve these problems, this paper proposes a novel intelligent method of condition diagnosis for plant machinery by using Relative Ratio Symptom Parameters (RRSPs) and Bayesian Network. The RRSPs in low- frequency area are defined for reflecting the feature of vibration signals measured in each state. Moreover, Discrimination Index (DI) has also been defined to evaluate the applicability of
RRSPs. Practical example of fault diagnosis for diagnosing structural faults of rotating machinery, such as misalignment unbalance and loose states which are often accruing in a rotating machine are shown to verify that the proposed method is effective $^{[5]}$.

\section{SYMPTOM PARAMETER AND}

\section{DISCRIMINATION INDEX}

In this paper ten new symptom parameters called "Relative Ratio Symptom Parameters (RRSPs)" in low- frequency area for diagnosing structural faults of rotating machinery, such as misalignment, loose and unbalance state, are defined and given as follows.

$$
R_{1}=\frac{A_{a d} / A_{r d}}{A_{a n} / A_{r n}}
$$

Here, $A_{a n}$ and $A_{r n}$ are the vibration level in shaft direction and radial direction in normal state respectively, $A_{a d}$ and $A_{r d}$ are the vibration level of shaft direction and radial direction in abnormal state respectively. (Vibration level is standard deviation or root mean square value .etc.)

$$
R_{2}=\frac{P_{d}\left(f_{r}\right) / P_{n}\left(f_{r}\right)}{\left|N_{d}-N_{n}\right| / 100}
$$

Here, $P_{n}\left(f_{r}\right)$ and $P_{d}\left(f_{r}\right)$ are the spectrum value at $f_{r}$ in normal state and abnormal state 
respectively, $N_{n}$ and $N_{d}$ are the rotation speed of the machine in normal state and abnormal state respectively.

$$
\begin{gathered}
R_{3}=\frac{P_{d}\left(f_{r}\right) / \sum_{i=2}^{20} P_{d}\left(i \cdot f_{r}\right)}{P_{n}\left(f_{r}\right) / \sum_{i=2}^{20}{ }_{n}\left(i \cdot f_{r}\right)} \\
R_{4}=\frac{P_{d}\left(2 f_{r}\right) / P_{d}\left(f_{r}\right)}{P_{n}\left(2 f_{r}\right) / P_{n}\left(f_{r}\right)} \\
R_{5}=\frac{P_{d}\left(3 f_{r}\right) / P_{d}\left(f_{r}\right)}{P_{n}\left(3 f_{r}\right) / P_{n}\left(f_{r}\right)} \\
R_{6}=\frac{\sum_{i=4}^{10} P_{d}\left(i \cdot f_{r}\right) / P_{d}\left(f_{r}\right)}{\sum_{i=4}^{10} P_{n}\left(i \cdot f_{r}\right) / P_{n}\left(f_{r}\right)}
\end{gathered}
$$

Here, $f_{r}$ is the rotating frequency. $P_{n}\left(i \cdot f_{r}\right)$ and $P_{d}\left(i \cdot f_{r}\right)$ are the high-order harmonic value at $i \cdot f_{r}$ in normal and abnormal state respectively.

$$
R_{7}=\frac{A_{v d} / A_{h d}}{A_{v n} / A_{h n}}
$$

Here, $A_{v n}$ and $A_{h n}$ are the vibration level in vertical direction and horizontal direction in normal state respectively, $A_{v d}$ and $A_{h d}$ are the vibration level of vertical direction and horizontal direction in abnormal state respectively.

$$
\begin{aligned}
R_{8}= & \frac{\sum_{>0.6 k H}^{<1.5 k H} P_{d}(f) / \sum_{<0.6 k H} P_{d}(f)}{\sum_{<0.6 k H}^{1.5 k H} P_{n}(f) / \sum_{<0.6 k H} P_{n}(f)} \\
R_{9}= & \beta_{d}-\beta_{n}
\end{aligned}
$$

Here, $\quad \beta_{n}$ and $\beta_{d}$ are the skewness in normal state and abnormal state respectively.

$$
R_{10}=\frac{\sum_{i \neq i \cdot f_{r}}^{f_{i}>6 f_{r}} P_{d}\left(f_{i}\right) / \sum_{i=1}^{6} P_{d}\left(i \cdot f_{r}\right)}{\sum_{i \neq i \cdot f_{r}}^{f_{i}>6 f_{r}} P_{n}\left(f_{i}\right) / \sum_{i=1}^{6} P_{n}\left(i \cdot f_{r}\right)}
$$

The Discrimination Index (DI) is defined to evaluate the diagnosing sensitivity of RRSP. The quality of a symptom parameter (SP), which will be used to distinguish two states, such as normal and abnormal state, is evaluated by Discrimination Rate (DR) and Discrimination Index (DI) ${ }^{[1]}$ [3]. Supposing that $\mathrm{x}_{1}$ and $\mathrm{x}_{2}$ are the SP values calculated from the signals measured in state 1 and state 2 respectively, and their average and standard are $\mu$ and $\sigma$. The DR and DI are calculated by

$$
\begin{aligned}
& D R=1-\frac{1}{\sqrt{2 \pi}} \int_{-\infty}^{-D I} \exp \left(-\frac{\mu^{2}}{2}\right) d_{\mu} \\
& D I=\frac{\left|\mu_{2}-\mu_{1}\right|}{\sqrt{\sigma_{1}{ }^{2}+\sigma_{2}{ }^{2}}} \text { or } D I=\frac{\left|\overline{x_{2}}-\overline{x_{1}}\right|}{\sqrt{\sigma_{1}{ }^{2}+\sigma_{2}{ }^{2}}}
\end{aligned}
$$

\section{BAYESIAN NETWORK}

Bayesian Network (BN) is a kind of probability network used to describe the relationship between variables, and it is also acyclic graph with probability label which can visually show the distribution function of joint probability between corresponding variables. Bayesian Network structural model can be combined by the network nodes (discrete or continuous), sets of directed edges which connect the network nodes with special relationships and that conditional probability is used to describe the interaction between nodes.

Bayesian Network(BNC) can be described in detail and clearly as follows: Bayesian Network is combined by $n$ nodes, expressed by $B(G, P)$, which is made of two parts:

(1) G is the structure of Bayesian Network, and it is also a directed acrylic graph showing the dependence and independence among the nodes. Every node in the graph is a random variable and the directed edges between nodes represent the relation of each other. Moreover, the nodes can be the abstract of any problems, such as diagnosis assumption、 test value, monitoring phenomenon, etc. Bayesian Network regulates that every node $\mathrm{X}_{\mathrm{i}}$ is independent with other nodes. That is, if $\mathrm{A}$ $\left(X_{i}\right)$ is sets of any nodes which is not filial generation of $\mathrm{X}_{\mathrm{i}}$. and $\mathrm{D}\left(\mathrm{X}_{\mathrm{i}}\right)$ is the direct parent nodes, then $I\left(X_{i}, A\left(X_{i}\right) / \pi\left(X_{i}\right)\right)$ means: $P\left(X_{i} / A\left(X_{i}\right), \pi\left(X_{i}\right)\right)=P\left(X_{i} / \pi\left(X_{i}\right)\right)$

(2) $\mathrm{P}$ is a conditional probability table of every node, depicting the dependence between nodes and their parent nodes rationally which can be written as $\mathrm{P}\left(\mathrm{X}_{\mathrm{i}} \mid \mathrm{D}\left(\mathrm{X}_{\mathrm{i}}\right)\right)$. The conditional probability of nodes without parent nodes is 
called prior probability. Based on the nodes, directed edges and conditional probability, Bayesian Network can be used to describe the joint probability between nodes. We can obtain the joint distribution by applying the conditional dependence in chain rules:.

$P(U)=P\left(X_{1}, X_{2}, . . X_{n}\right)=\prod^{n} P\left(X_{i} / \pi\left(X_{i}\right)\right)$

Furthermore, the joint probability distribution of the variables in the following graph can arrive ${ }^{\text {at: }} P\left(X_{1}, X_{2}, \ldots, X_{6}\right)=\prod_{i=1}^{6} P\left(X_{i} / \pi\left(X_{i}\right)\right)$

$=P\left(X_{1}\right) P\left(X_{2} / X_{1}\right) P\left(X_{3} / X_{1}\right) P\left(X_{4} X_{2}\right) P\left(X_{5} / X_{2}, X_{3}\right) P\left(X_{6} X_{5}\right)$ According to the above analysis, we know Bayesian Network is kind of decomposed expression of joint probability distribution that is Bayesian Network can simplify the joint probability of variables.

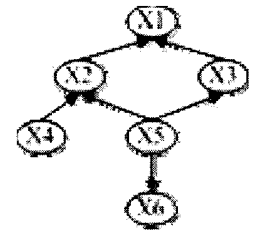

Figure 1. General model of Bayesian Network for fault diagnosis

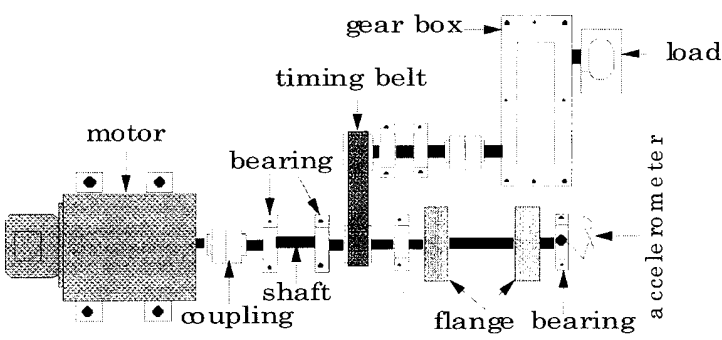

Figure2. Experimental equipment for fault diagnosis

\section{4 . BAYESIAN NETWORK MODEL FOR}

\section{ROTATING MACHINERY DIAGNOSIS}

\section{A. Rotating machine for fault diagnosis}

The rotating machine for the diagnosis is shown in Fig.2. The subject of the diagnosis is to distinguish shaft misalignment, unbalance and loose state, which are called "structural faults on rotating machine" and are often occurring in rotating machinery. The accelerometer is used to measure vibration signals of the rotating machine. The sampling frequency of signal measurement is
$5000 \mathrm{H}_{\mathrm{Z}}$, and the sampling time is $20 \mathrm{~s}$. Raw vibration signals in the normal, misalignment, unbalance and loose states are shown in Figure.3, respectively.

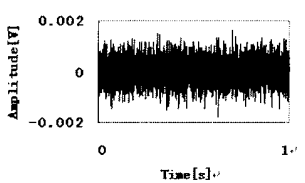

(a)normal state

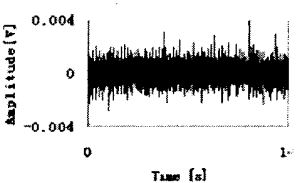

(c)misalignment state

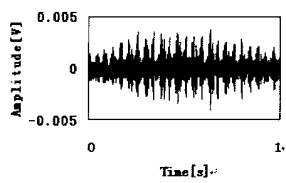

(b) loose state

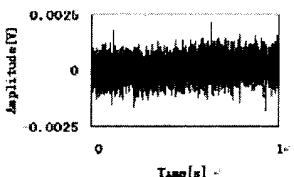

(d) unbalance state
Figure 3.Original vibration signals of all states

\section{B. Establishment and verification of Bayesian}

Network in the fault diagnosis

In this paper the five Bayesian Network models for the condition diagnosis based on Bayesian Network theory are constructed, and through the comparison of the diagnosis results, the most suitable model of Bayesian Network for the condition diagnosis is selected.

Figure 6-8 show the Bayesian Network model 1-3 for the condition diagnosis respectively, which consist of four layers. The first layer is normal state and abnormal state. The second layer is combinations of misalignment, unbalance and loose states. The third layer is misalignment, unbalance and loose states, and the last layer is ten Relative Ratio Symptom Parameters (RRSPs) calculated by formulae 1-10 and the signals shown in Figure 3, from which the possibility grades of each state can be obtained.

Table1-4 show the names and values of each layer node respectively.

Table1. The names and values of the first layer

\begin{tabular}{|c|l|}
\hline State & \multicolumn{1}{|c|}{ Values of each state } \\
\hline Normal state (N) & $\begin{array}{l}0,1 \\
(0: \text { abnormal; } 1: \text { normal })\end{array}$ \\
\hline Abnormal state (UN) & $\begin{array}{l}0,1 \\
(0: \text { normal; } 1: \text { abnormal })\end{array}$ \\
\hline
\end{tabular}


Table2. The names and values of the second layer

\begin{tabular}{|c|c|l|}
\hline & Fault states & \multicolumn{1}{|c|}{ Values of each state } \\
\hline $\begin{array}{c}\text { Fault } \\
\text { events } \\
\text { layer }\end{array}$ & unbalance (U) & $\begin{array}{l}0,1 \\
(0: \text { normal; } 1 \text { : fault })\end{array}$ \\
\cline { 2 - 3 } & & $\begin{array}{l}0,1 \\
(0: \text { normal; } 1 \text { : fault })\end{array}$ \\
\cline { 2 - 3 } & misalignment (M) & $\begin{array}{l}0,1 \\
(0: \text { normal; } 1 \text { : fault })\end{array}$ \\
\cline { 2 - 3 } & loose (L) & \\
\end{tabular}

Table3. The names and values of the third layer

\begin{tabular}{|c|c|c|}
\hline & Fault states & Values of each state \\
\hline \multirow[t]{3}{*}{$\begin{array}{c}\text { Fault } \\
\text { events } \\
\text { layer }\end{array}$} & $\begin{array}{l}\text { Unbalance or } \\
\text { misalignment } \\
\text { ( } \mathrm{U} \text { or } \mathrm{M})\end{array}$ & $\begin{array}{l}\quad 0,1 \\
(0: \text { loose; } \\
1: \text { unbalance } \\
\text { or misalignment })\end{array}$ \\
\hline & $\begin{array}{l}\text { Misalignment } \\
\text { or loose } \\
(\mathrm{M} \text { or } \mathrm{L})\end{array}$ & \begin{tabular}{l}
\multicolumn{1}{c}{0,1} \\
$(0:$ unbalance; \\
$1:$ Misalignment or \\
loose $)$
\end{tabular} \\
\hline & $\begin{array}{l}\text { Unbalance or loose } \\
\text { (U or } \mathrm{L})\end{array}$ & $\begin{array}{l}\text { } 0,1 \\
(0: \text { Misalignment; } \\
1: \text { unbalance or } \\
\text { loose })\end{array}$ \\
\hline
\end{tabular}

Table4. The names and values of the last layer

\begin{tabular}{|c|c|c|}
\hline \multirow{4}{*}{$\begin{array}{c}\text { Symptom } \\
\text { parameters layer }\end{array}$} & $\begin{array}{c}\text { Symptom } \\
\text { parameters }\end{array}$ & \multicolumn{2}{|c|}{ Values } \\
\cline { 2 - 3 } & $\mathrm{R}_{1}$ & $1,2,3,4,5$ \\
\cline { 2 - 3 } & $\mathrm{R}_{2}$ & $1,2,3,4,5$ \\
\cline { 2 - 3 } & $\mathrm{R}_{3}$ & $1,2,3,4,5$ \\
\cline { 2 - 3 } & $\mathrm{R}_{4}$ & $1,2,3,4,5$ \\
\cline { 2 - 3 } & $\mathrm{R}_{5}$ & $1,2,3,4,5$ \\
\cline { 2 - 3 } & $\mathrm{R}_{6}$ & $1,2,3,4,5$ \\
\cline { 2 - 3 } & $\mathrm{R}_{7}$ & $1,2,3,4,5$ \\
\cline { 2 - 3 } & $\mathrm{R}_{8}$ & $1,2,3,4,5$ \\
\cline { 2 - 3 } & $\mathrm{R}_{9}$ & $1,2,3,4,5$ \\
\cline { 2 - 3 } & $\mathrm{R}_{10}$ & 2,5 \\
\cline { 2 - 3 }
\end{tabular}

Note, $1:$ very small; $2:$ small; $3:$ middle; $4:$ big; $5:$ very big

Besides, it is very important to make sure the possible relationship between parent nodes and nodes before learning of Bayesian fault diagnosis Network. The following table lists several kinds of possible relationships. The first line in Table 5 is the nodes in the Bayesian Network including the parent nodes and filial nodes, the rear lines are the corresponding parent nodes and filial nodes in which the nodes with * are necessary, while the nodes without $*$ are possible, However, the final results are determined by the learning and inference of Bayesian Network. On the basis of the above relationship, Bayesian fault diagnosis Network Model is built, as in Fig 6 9. By dividing the vibration signals measured in the experiment into two groups, one is used to input into the model 1-4 for learning and inference, and another group for verifying the effectiveness of Bayesian Network in the condition diagnosis. The inference results of 4 models for the condition diagnosis are shown in table 6 . The values in the table 6 are the probabilities which express the correct rates of distinguishing each state by the Bayesian Network.

Table5. Relationship between nodes (a) For model 1

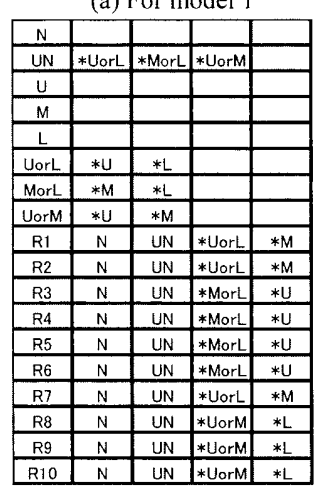

(b) For model 2

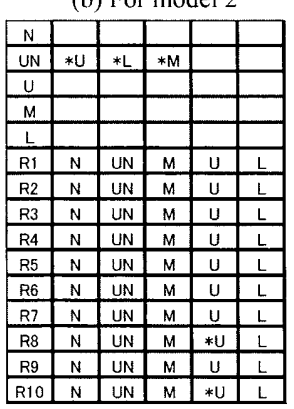

(c) For model 3

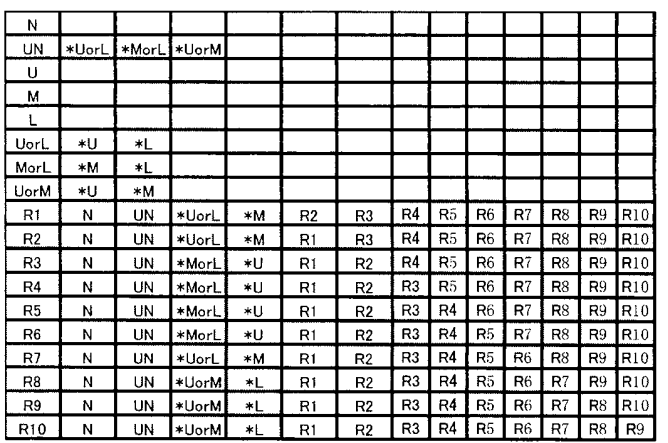

(d) For model 4

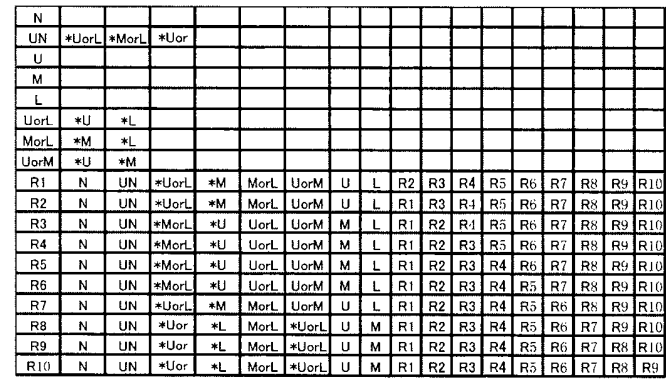



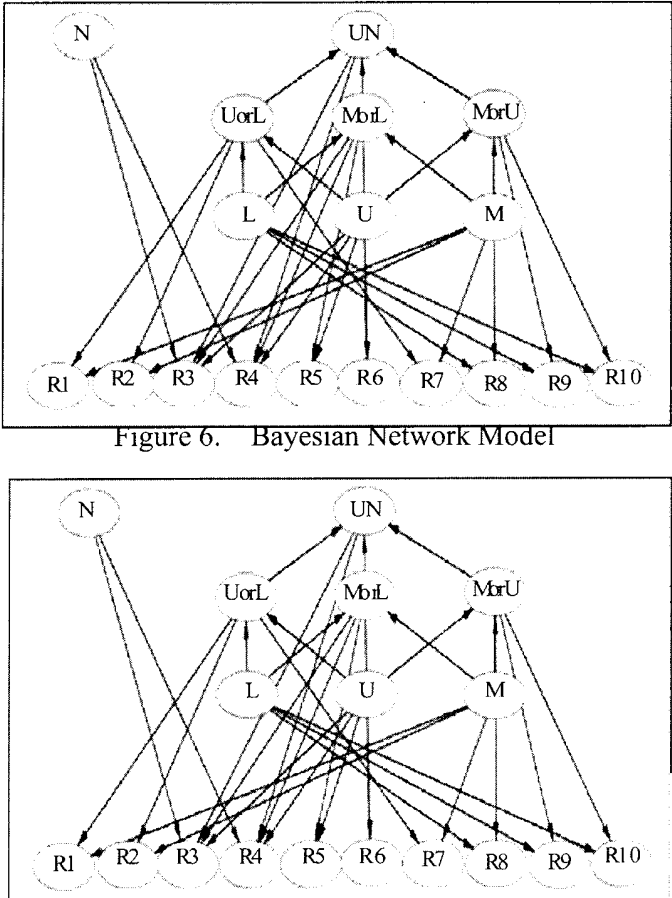

Figure 7. Bayesian Network Model 2

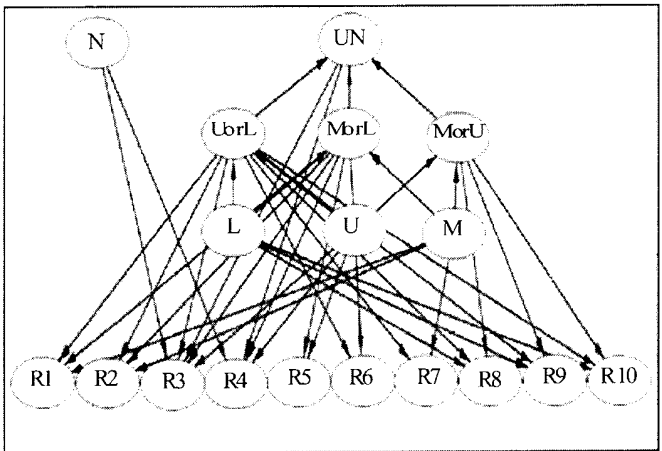

Figure 8. Bayesian Network Model 3

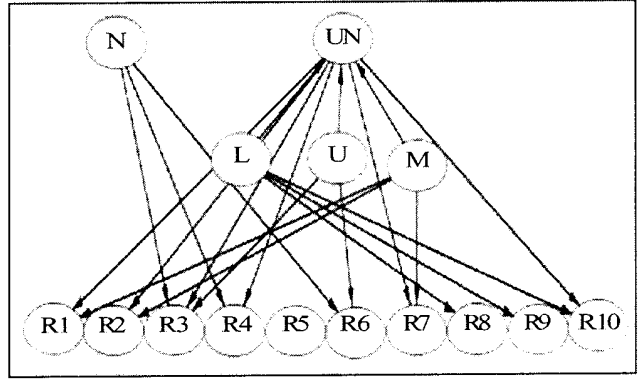

Figure 9. Bayesian Network Model 4
Table 6.The diagnosis results of model 1-4

\begin{tabular}{|c|c|c|c|c|}
\hline & $\begin{array}{c}\text { Model } \\
1\end{array}$ & $\begin{array}{c}\text { Model } \\
2\end{array}$ & $\begin{array}{c}\text { Model } \\
3\end{array}$ & $\begin{array}{c}\text { Model } \\
4\end{array}$ \\
\hline $\begin{array}{c}\text { Probability of } \\
\text { normal state }\end{array}$ & 0.6634 & 0.6589 & 0.6754 & 0.6984 \\
\hline $\begin{array}{c}\text { Probability of } \\
\text { misalignment } \\
\text { state }\end{array}$ & 0.9982 & 0.9994 & 0.9999 & 0.9472 \\
\hline $\begin{array}{c}\text { Probability of } \\
\text { loose state }\end{array}$ & 0.9990 & 0.9991 & 0.9983 & 0.9994 \\
\hline $\begin{array}{c}\text { Probability of } \\
\text { unbalance state }\end{array}$ & 0.0229 & 0.2460 & 0.3332 & 0.2169 \\
\hline
\end{tabular}

From the above table, we know the probability of normal state of model $1-4$ is near 0.7 . The probability of unbalance state is near 0.3 , and probabilities of misalignment and loose state are near 1. The results in table 6 show that ten symptom parameters (RRSPs) shown in formulae (1)-(10) are suitable for effectively distinguishing normal state and tow faults (misalignment state and loose state). However, because the probability of unbalance state is not high, the symptom parameters inputted into the Bayesian Network should be analyzed and selected by DI shown in formula (12), in order to raise the diagnosis sensitivity of unbalance state. The model 4 is improved to model 5 .

Table 7 lists the DI of each RRSP for normal state and abnormal state, and loose state, misalignment state and unbalance state calculated using formulae (1)-(10) and the signals shown in Figure 3.

Table 8 lists the RRSPs selected on the basis of the DIs. Since all of DIs are larger than 2.9, all of the detection rates (DR) are larger than $99 \%$.

By the same verification method as used in model 1-4, the test data and the relationship between nodes were input in the learned Bayesian Network model 5, and the Bayesian Network correctly and quickly judged the states. The diagnosis results show that the probability of normal state, misalignment, loose and unbalance state are $0.9601,0.9603, \quad 0.9999, \quad 0.5930$ respectively. The accuracy is greatly improved. 
Table 7 DI of RRSP for each state

\begin{tabular}{|c|c|c|c|c|}
\hline RRSP & $\mathrm{DI}_{\left(\mathrm{N}^{-} \mathrm{UN}\right)}$ & $\mathrm{DI}_{(\mathrm{M}-\mathrm{L})}$ & $\mathrm{DI}_{(\mathrm{M}-\mathrm{U})}$ & $\mathrm{DI}_{(\mathrm{L}-\mathrm{U})}$ \\
\hline $\mathrm{R} 1$ & 2.66 & 3.28 & 0.05 & 3.16 \\
\hline $\mathrm{R} 2$ & 5.00 & 5.01 & 0.36 & 5.01 \\
\hline $\mathrm{R} 3$ & 1.04 & 1.71 & 1.80 & 0.30 \\
\hline R4 & 0.05 & 1.22 & 0.46 & 1.50 \\
\hline R5 & 0.02 & 0.62 & 0.62 & 0.01 \\
\hline R6 & 0.10 & 1.18 & 1.29 & 0.30 \\
\hline R7 & 1.41 & 1.39 & 0.02 & 1.39 \\
\hline R8 & 0.61 & 2.15 & 1.05 & 2.91 \\
\hline R9 & 0.03 & 0.12 & 0.89 & 0.07 \\
\hline R10 & 2.70 & 3.16 & 3.81 & 0.69 \\
\hline
\end{tabular}

Table 8.The RRSP for each state

\begin{tabular}{|c|c|}
\hline State & Symptom parameter \\
\hline Normal state & $\mathrm{R}_{1}, \mathrm{R}_{2}, \mathrm{R}_{10}$ \\
\hline Misalignment state & $\mathrm{R}_{1}, \mathrm{R}_{2}, \mathrm{R}_{10}$ \\
\hline Loose state & $\mathrm{R}_{1}, \mathrm{R}_{2}, \mathrm{R}_{8}, \mathrm{R}_{10}$ \\
\hline Unbalance state & $\mathrm{R}_{1}, \mathrm{R}_{2}, \mathrm{R}_{8}, \mathrm{R}_{10}$ \\
\hline
\end{tabular}

Table 9.The relationship between nodes for model 5

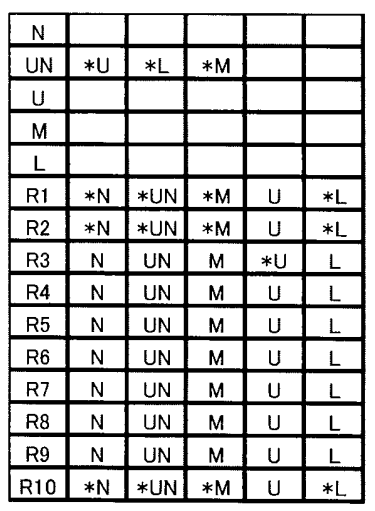

\section{CONCLUSIONS}

In order to improve the efficiency of the condition diagnosis for plant rotating machinery and distinguishing fault types at an early stage, this paper proposed a novel intelligent condition diagnosis method for rotating machinery using Relative Ratio Symptom Parameters (RRSPs) and Bayesian Network, by which the state of machinery can be automatically judged on the basis of the possibility grades of normal state and each abnormal state. The ten RRSPs in lowfrequency area were defined, which can reflect the characteristics of time signal measured for the fault diagnosis of rotating machinery. The Discrimination Index (DI) was also used to evaluate the sensitivity of RRSPs for detecting and distinguishing faults. Moreover, in this paper five Bayesian Network models for the condition diagnosis are constructed, and through the practical example of diagnosis of rotating machinery for detecting and distinguishing structural faults states, such as misalignment unbalance and loose states which often occur in rotating machinery, the most suitable model of Bayesian Network for the condition diagnosis was decided.

\section{REFERENCE}

[1] Peng Chen, Toshio Toyota and Zhengja He, Automated Function Generation of Symptom Parameters and Application to Fault Diagnosis of Machinery under Variable Operating conditions; IEEE TRANSACTIONS ON SYSTEMS ' 01 . No.6, p.775-781

[2] P. CHEN, T. TOYOTA, Fuzzy Diagnosis and Fuzzy Navigation for Plant Inspection and Diagnosis Robot, Proc. of FUZZ-IEEE/IFES'95, Vol.1, p. 185-193.

[3] H. Wang, P. Chen, Condition diagnosis of blower system using rough sets and a fuzzy neural network, WSEAS TRANSACTIONS ON BUSINESS AND ECONOMICS'08, Vol. 5, Issue 4, p. 58-63.

[4] H. Matuyama, Diagnosis Algorithm, JOURNAL OF JSPE, Vol.75, 1991.No.3, p.35-37.

[5] Jianghua Luo. The application of Bayesian Network in the mechanical fault diagnosis, Chongqing University [D],2006.

[6] Songqing Peng, Yousheng Zhang. Research on the theory of Bayesian Network and Its implication in Image Analysis. Hefei industrial university[D],2005

[7] Jianchuan Li, Huniao Qing,etc. Bayesian Network theory in the equipment fault device, CHINA MECHANICAL ENGINEERT, Vol.10,2003

[8] Wilson Quansheng Wang, Rotary machinery health condition monitoring, fault diagnosis [D], Waterloo University, 2002 